\title{
Della luce e della visibilità \\ Considerazioni in margine all'opera di Vincenzo Consolo
}

\section{Paola Capponi}

Universidad de Sevilla

\begin{abstract}
La luce, la luminosità, ossia le forme con cui la luce è presente nell'intertesto consoliano paiono assumere valenze che trascendono la mera descrittività, la nota paesaggistica.

Un lessico della luce (e dell'ombra) preciso e puntuale definisce il rapporto tra Milano e la Sicilia, tra il centro e la periferia, tra passato e presente. L'intreccio di tali coppie oppositive rende incalzanti i rimandi dal piano diatopico (Milano-Sicilia) a quello diacronico (passato-presente). La riflessione stessa sulla memoria e sulla possibilità del narrare attinge ad un repertorio di immagini e di luoghi della tradizione che si rifanno alla luce come elemento metaforico portante. Si apre così la possibilità di articolare intorno al tema della luce un percorso che, attraverso più livelli di lettura, arriva ad alcuni nodi della scrittura dell'autore.
\end{abstract}

Parole chiave: luce, lessico, diatopia, diacronia, memoria (Vincenzo Consolo).

\section{Abstract}

The light, the brightness, namely the shapes in which light is present throughout Consolo's writing seem to take on contents that transcend mere descriptiveness and notes on the landscape.

A precise and punctual vocabulary of light (and shadow) defines the relationship between Milan and Sicily, between the centre and the periphery, between past and present. The interweaving of such opposing pairs makes the references from the diatopic plane (Milan-Sicily) to the diachronic plane (past-present) more imminent. The reflection on memory itself and on the ability to tell a story draws on a repertoire of images and traditional places that take their revenge on light as the main metaphorical element. This opens up the possibility of constructing a path around the subject of light, which, on several levels of reading, gets to the heart of the author's writing.

Key words: light, vocabulary, diatopics, diachronics, memory (Vincenzo Consolo). 
1.

Per motivare la scelta della luce come oggetto di riflessione, parto da un testo breve, 29 aprile 1994: cronaca di una giornata (pubblicato nel numero 29 di Nuove Effemeridi) in cui Vincenzo Consolo racconta di una mattinata dedicata alla scrittura (e precisamente alla stesura de L'olivo e l'olivastro) e alla memoria, al flusso memoriale, interrotta a mezzogiorno per comprare il giornale, grigio momento in cui rientrare nell'avvilente e bigia quotidianità. La cesura della giornata, la bipartizione, evidente e chiara nella brevità del testo nonché le riflessioni iniziali, proprie del momento della scrittura, della narrazione e della memoria, offrono lo spunto per alcune osservazioni di carattere più generale che costituiscono la nervatura di questo intervento.

Due sono gli elementi sui quali voglio soffermarmi: la cesura e il viaggio. Cesura, ossia spaccatura, individuazione di due poli distinti, e viaggio, quasi geometricamente inteso, quale tratto, segmento che unisce due punti separati.

Nella prima parte del testo, Consolo racconta di un viaggio, anzi di molti viaggi e, più propriamente, di fughe, allontanamenti in cui punto di dipartita e punto d'approdo si intrecciano, alternandosi. I due poli sono la Sicilia e Milano; al movimento di fuga dalla Sicilia, dalla periferia verso un Nord di lavoro, di rispetto di leggi e di diritti, al viaggio da una terra di «immobilità, privazione e offesa» ${ }^{1}$ verso la città dei lumi, si oppone un movimento contrario che dalla città delle nebbie riporta all'isola del sole.

Vado in Sicilia e ne fuggo ogni volta, ritorno a Milano [...] Credo sia questo ormai il destino d'ogni ulisside d'oggi, di tornare sovente nell'Itaca del distacco e della memoria e di fuggirne ogni volta, di restare prigioniero nella reggia d'Alcinoo, in quel regno di supposta utopia, d'irreale armonia, condannato a narrare all'infinito, come un cieco cantore, un vecchio svanito, i suoi nosto $i$, le sue odissee. ${ }^{2}$

Il nostos fiorisce e si complica dunque in una serie di viaggi senza posa, in cui la Sicilia già non è più l'isola del Sud, geograficamente e puntualmente individuabile, ma l'Itaca dei distacchi e dei ritorni infiniti, la terra cui si anela tornare e da cui si continua a fuggire, ormai spaccata, franta in lacerazioni amare. Il nostos privato si fa viaggio di "ogni ulisside d'oggi», viaggio che presuppone un ritorno o, meglio, un movimento pendolare perpetuo, quasi tra $\mathrm{i}$ labbri di una ferita, tra i lembi di una frattura lenita solo dalla narrazione.

Proprio la biforcazione è eletta a simbolo e titolo in L'olivo e l'olivastro. L'opposizione coltivato-selvatico, civile-barbarico non è solo emblema della condizione della Sicilia, bensì di una regione universale, di una Sicilia che si fa «metafora dell'Italia (dell'Europa, del mondo?)». ${ }^{3}$ La frattura è certo quella

1. Vincenzo Consolo, «29 aprile 1994: cronaca di una giornata», in Nuove Effemeridi, n. 29, 1995 , p. 4.

2. Ibid., p. 4.

3. Ibid., p. 5. 
tra la città del Nord, Milano, e l'isola del Sud, la Sicilia, ma è anche spaccatura interna alla Sicilia stessa, terra dal glorioso e sfavillante passato e dal presente oscurato da privazioni e barbarie, ed è, in ultima analisi, condizione comune, universale.

Se in una dimensione sincronica, possiamo rintracciare dunque l'opposizione tra Milano e Sicilia, centro e periferia, luce e ombra, e ricostruire geograficamente il viaggio tra i due poli, nella dimensione diacronica individuiamo il contrasto tra la luce del passato e il buio del presente.

Si intrecciano insomma due coppie oppositive, una sul piano orizzontale, diatopico: [Milano : Sicilia] e una su quello verticale, diacronico: [Presente : Passato]. La combinazione dei due piani è una delle cifre della scrittura consoliana: i tagli diacronici sono abilmente incastonati nella tessitura lineare, quasi incursioni verticali che paiono imprimere alla scrittura un movimento vorticoso, un avvitamento che penetra le stratificazioni della storia e acuisce il «dolore» della lacerazione. Il viaggio non è tanto rappresentabile come linea piana che unisce due punti, quanto come percorso carsico, a volte riaffiorante in superficie, trivellazione di strati profondi, discesa nelle profondità ipogee.

A Siracusa stride il contrasto tra lo sfarzo della potenza antica e il degrado presente:

Mi trovo, confuso, smarrito, in questo teatro di profonda memoria, di continui richiami, in questa scena odierna di degrado e macerie, deserta di parola, poesia, in questa cavea di urla e fragori, sotto un cielo di spesse caligini, presso un mare di petroli e liquami. In Siracusa è scritta la storia dell'infinito tramonto della civiltà nostra e cultura, dell'umano sentire, è impressa la notte della ragione e della pietà. ${ }^{4}$

A Milano non meno doloroso è lo spegnersi dei Lumi, un tempo baluardo e faro per la società civile, ora fioche luci tra le nebbie:

questa città del disinganno, dell'utopia crollata, della mediocrità più squallida, della nevrosi e dell'aggressività, del deserto d'ogni gioia, d'ogni bellezza, perché non c'è più un'Itaca dove ridurmi e conciliarmi, in cui ricomporre l'armonia perduta. ${ }^{5}$

La desolazione del tempo presente irrompe nella giornata di Consolo quando, a mezzogiorno, sospende il lavoro (e, con esso, il fluire di antiche memorie) e compra il giornale: «È il momento, quello, della frattura, del ritorno brusco nella prosa offensiva del presente, dell'ingresso nel grigio miserevole teatro di questo regno dei Feaci». ${ }^{6}$ La chiusura del testo è affidata ad un'immagine di allargato tramonto infinito, che cala sulla giornata (il 29 aprile 1994), su Siracusa e su Milano: "Tutto ormai in questo Paese è di banalità e orrore, 
di degrado e oblìo, è tramonto infinito, è Siracusa, è fiammella d'olio o di candela che si spegne, è buio di catacomba». ${ }^{7}$ Lenizione e riparo all'offesa presente è l'interruzione stessa del presente, la sua sospensione, e quindi lo scrivere e la memoria: "Mai sempre tuttavia il viaggio, come distacco, come lontananza dalla realtà che ci appartiene è un sognare. E sognare è vieppiù lo scrivere, lo scriver memorando del passato come sospensione del presente, del viver quotidiano. E un sognare infine, in suprema forma, è lo scriver d'un viaggio, e d'un viaggio nella terra del passato». 8

\section{2.}

La luce è elemento fondante del panorama meridionale. La luminosità e la pienezza di luce sono dati del paesaggio, tratto distintivo della campagna siciliana arsa, bruciata dal sole, ostica, assopita nei silenzi meridiani («Nei silenzi, si udiva solo il mormorìo dei piccioni, il lamento della cicala, di tutta la campagna sotto il sole» ${ }^{9}$ ), vibrante nell'aria calda del meriggio:

il sole [...] dal balcone era arrivato prima sulla tovaglia. Così caldo, questo sole, che si levava già dalla rena alla marina il fumo tremolante del vapore. [...]

Due cani si mordevano gli orecchi saltando sopra il mucchio dei rifiuti, si davano zampate, giravano attaccati attorno a un palo inesistente. Questo sole. ${ }^{10}$

La Sicilia si presenta tutta «sotto il segno del sole», terra dura di stenti e di fatiche, di uomini «nutriti di sarde e di cicorie ed asciugati al sole», ${ }^{11}$ che mangiano «sole come pane». ${ }^{12}$

La luce riflessa sulle pietre, nel riverbero del mare, battente sugli splendori antichi di antiche città, è sfarzo e bagliore accecanti; filtra, rimbalza tra le mura e i palazzi, le rovine e i ruderi, quasi principio vivificante in grado di restituire in forma di miraggio l'antico splendore, grazia e armonia del passato. L'opposizione è netta rispetto alle città del Nord, rispetto a Milano, città di brume e di foschie, di luci spente:

una terra nordica, luntana, 'na piana chiusa da montagne altissime, d'eterni ghiacci e d'intricati boschi, rotta da lunghi fiumi e laghi vasti, terra priva di mare, cielo, sole, stelle, lune, coi verni interminabili carichi di nevi, e con le stati brevi, umide, brumose, ove la gente ognora mangia lardi, cotiche, verze, ranocchi, passeri, pulenta di granturco... ${ }^{13}$

7. Ibid., p. 7.

8. ID., Retablo, Palermo: Sellerio, 1987, p. 77.

9. ID., La ferita dell'Aprile, Milano: Mondadori, 1963, p. 147.

10. Ibid., p. 120.

11. Ibid., p. 106.

12. Ibid., p. 72.

13. Retablo, p. 66. 
La gamma cromatica calda dei tramonti siciliani, oro-arancio-viola, o lo sfavillío dorato dell'aurora a Palermo, "latteggiante e rosseggiante», stride con le sfumature nel grigio delle nebbie e dei fumi, con il biancastro e con il grigiastro del gelo del Nord. Nell'ora del tramonto, l'occhio indugia nel paesaggio di Sicilia mutante di colori; un sole cefalutano «imporporava il mondo, stramangiava le cose, faceva di fuoco cielo, terra alberi, uomini, rendeva irreale ogni presenza, movimento». ${ }^{14}$

E un paesaggio in vitro quello offerto dalla città dei Lumi, da cui si fugge, di desolante grigia uniformità, indefinitezza di confini in cui vaga l'occhio vacuo e perso:

Era una mattina di novembre. Al di là del capannone, oltre i vetri polverosi, si scorgeva un piccolo campo, un terreno vago circondato d'altri fabbricati, d'altri capannoni con ciminiere, sfumati dalla nebbia, un campo sulle cui stoppie marcite di granturco gravava una bruma grigiastra, si stendeva una pellicola biancastra di brina gelata. ${ }^{15}$

La terra a cui tornare, l'armonia perduta cui ricongiungersi richiamata quasi in controcanto dal panorama milanese è la Sicilia ma, ancora una volta, con drammatico taglio diacronico, non già la Sicilia d'oggi, quanto la Sicilia, metaforicamente intesa, d'un tempo:

la Sicilia era un'isola surreale o metafisica, con solo città sepolte, necropoli, latomie, ipogei pieni di sarcofagi di marmo luminoso, di neri lucidi crateri con dèi ed eroi graffiti; con templi teatri agorai di città morte in luoghi remoti, deserti, incontaminati. ${ }^{16}$

In una menzione del nostos di Ulisse (più volte echeggiato nei romanzi consoliani) offerta in L'olivo e l'olivastro è possibile rintracciare, esplicitato, l'intreccio delle due dimensioni. Così è raccontato il ritorno di Ulisse, dalle macerie di Ilio ad Itaca «chiara nel sole...» ${ }^{17}$ :

Viaggio da oriente verso occidente, in una dimensione orizzontale. Ma, una volta immerso nella vastità del mare, è come fosse il suo un viaggio in verticale, una discesa negli abissi, nelle ignote dimore, dove, a grado a grado, tutto diventa orrifico, subdolo, distruttivo. Si muove il navigante tra streghe, giganti, mostri impensati, tra smarrimenti, inganni, oblii, malie, perdite tremende, fino alla solitudine, all'assoluta nudità, al rischio estremo per la ragione e per la vita. ${ }^{18}$

14. ID., Nottetempo, casa per casa, Milano: Mondadori, 1992, p. 21.

15. ID., Le pietre di Pantalica, Milano, Mondadori, 1988, p. 112.

16. Ibid., p. 113.

17. ID., L'olivo e l'olivastro, Milano: Mondadori, 1994, p. 19.

18. Ibid., p. 19. 
Il ritorno pare coincidere qui con l'inabissamento di sé, con l'immersione nella memoria, con un percorso di progressivo avvicinamento al limen, al limite estremo, oltre il quale dilagano il silenzio e l'assenza. Procede il navigante (come la scrittura consoliana) per accumulazione, nel suo duplice percorso in orizzontale e in verticale; le due forze paiono comporsi in un movimento vorticoso, in un turbinío (o in una "chiocciola») che pare sottendere un unico punto di fuga, un occhio del ciclone in cui punto di dipartita e punto d'arrivo coincidano, ma la meta è solo supposta, suggerita, sfiorata e il ciclone pare sospeso a mezz'aria, pare progressivamente stringere le maglie intorno all'innominabile, come a stringere d'assedio il non detto o il non dicibile, il silenzio e l'assenza, in un abile e meticoloso lavorío di approssimazione.

L'Itaca con cui riconciliarsi è svanita, la terra patria cui è sempre dolce tornare è un luogo della memoria, una meta nel tempo. Il ritorno è ad un presente di degrado e di macerie, ombra della luce di un tempo, fantasma dell'armonia perduta. Nel cielo, le incrinature di fumo dei comignoli dell'Etna, parte del paesaggio antico di Sicilia, lasciano spazio ai fumi delle raffinerie, ai gas dei lacrimogeni, segno, nella volta celeste, delle crepe nella natura e nella società. Forma parte del paesaggio di Sicilia «la colonna attorta del fumo del cratere» ${ }^{19}$ dell'Etna, che si conficca, quasi incastonata, nel piano orizzontale del cielo, ma alle striature di fumi naturali e di nuvole si aggiungono e si sovrappongono, nel presente, i fumi del degrado, i miasmi della corruzione. A Milazzo, «la colonna di denso fumo» che si vede «levarsi fino al cielo» ${ }^{20}$ è il segno dell'esplosione della raffineria; "nel cielo si formano nuvole» ${ }^{21}$ quando a Comiso sono sparati lacrimogeni per disperdere la folla, mentre a Palermo "ristagna sulla città, come un'enorme nuvola compatta, il fumo dei rifiuti». ${ }^{22}$

Il sole, prima vivificante in un paesaggio armonico, ora secca i gelsomini, al sole di luglio s'incrosta e annerisce il sangue. ${ }^{23}$ La luce è elemento che pare acuire di forma tagliente la desolazione e il degrado; svela piazze svuotate, «vuoti gusci di cicale»; è stasi, paralisi, immobilità. A Comiso il sole illumina una città morta:

Non resterà di noi neanche una vuota, dorata carcassa, come quella della cicala scoppiata nella luce d'agosto. Non resterà compagna, figlio o amico; ricordo, memoria; libro, parola. ${ }^{24}$

Palermo «è fetida e infetta» nel «luglio fervido». Di Avola, «il nuovo paese ricostruito al piano dopo il terremoto" sono ricordate le perfette geometrie, la bellezza dell'antica architettura da cui il paese pare trarre "giustizia e armonia»; 
ne sono citate la laboriosità, l'attività umana fervente di dibattiti e di discussioni; è descritto un paese che, nomina omina, è "Apicola soave e laboriosa»:

Avola del terreno arso, del mandorlo, dell'ulivo, del carrubo, della guerra con il sole, con la pietra, la città nuova di geometrica armonia, di vie diritte, d'ariose piazze, d'architettura di luce e fantasia [...]. La vasta piazza quadrata, il centro del quadrato inscritto nell'esagono, [...] fu sempre il teatro di ogni incontro, convegno, assemblea, dibattito civile. ${ }^{25}$

L'armonia passata in cui la mente e la memoria trovano ristoro si frange nel presente, nel momento del «ritorno brusco nella prosa offensiva del presente»: 26

Entra nel vasto spazio nell'ora della luce umana, della calura che si smorza, nel meriggio tardo ch'era in passato del brulichìo, del brusìo sulla piazza [...] sotto il cielo fitto dei voli obliqui, degli stridi, dei rintocchi di San Nicola, di Santa Vénera, dell'Annunziata, che ora è vuota, deserta, sfollata come per epidemia o guerra [...]. Cos'è successo in questa vasta, solare piazza d'Avola? Cos'è successo nella piazza di Nicosia, di Scicli, Ispica, Modica, Noto, Palazzolo, Ferla, Floridia, Ibla? Cos'è successo in tutte le belle piazze di Sicilia, nelle piazze di quest'Italia d'assenza, ansia, di nuovo metafisiche, invase dalla notte, dalle nebbie, dai lucori elettronici dei video della morte?27

Alla luce e al caldo soffocanti, alle vuote ore meridiane, sarebbe dovuta seguire «l'ora della luce umana», che propizia e accompagna il dibattito, l'incontro, l'attività, ma il tramonto non raccoglie più oggi, come era nel passato, il fervore e l'azione, bensì abbandoni, vacuità, silenzi, attese.

3.

Con puntualità è rilevato il trascolorare della luce, modulazioni di intensità luminosa che accompagnano il procedere delle ore e scandiscono l'ordine delle attività umane. Il sole ritma inesorabilmente il tempo delle opere e i giorni, dei lavori della campagna e del mare: le ore mattutine sono le più adatte al lavoro, temibile è l'ora meridiana, con il sole a perpendicolo, mentre il tramonto coincide con la fine delle fatiche del giorno. E un tempo ciclico, chiuso nell'alternarsi di notte e dì. La luce è dapprima tenue, non ancora dispiegata:

Il paese, già sul mattino primo e nella luce ancor non dispiegata, era una scorrerìa di carri e bestie, di comitive allegre, di musicanti, villani e mercanti che per ogni porta, di Trapani e Palermo, dello Stellario, di Corleone e del Castello, invadevano le strade e le piazze. ${ }^{28}$

25. L'olivo e l'olivastro, p. 110.

26. 29 aprile $1994 \ldots$, p. 6.

27. L'olivo e l'olivastro, p. 112.

28. Retablo, p. 50. 
poi si fa luce ferina, nel meriggio:

Dall'alba dava forte con la sua sciamarra, un colpo dietro l'altro, rantolando, hah hah, su quella crosta dura di petraia, in dorso di collina declinante, pane tomazzo e acqua unico ristoro a mezzogiorno. Piegato in due. Zuppa la camicia e il gilè, il fazzoletto al collo, con quel sole di maggio che ancora gli mordeva sulle spalle. ${ }^{29}$

ed è infine luce rosseggiante, prima di spegnersi al tramonto:

Il sole sbucava all'orizzonte sotto una banda nera di foschìa, e prima d'eclissarsi dietro i monti, dietro Barrafranca, Pietraperzìa, rosseggiò potente, infiammò gli uomini sopra Ratumeni, le pietre bianche della masseria. Poi, lentamente tramontò e tutto s'incupì. Smisero i braccianti d'arare, di zappare ed erpicare, riposero gli attrezzi. ${ }^{30}$

La scomparsa del sole dietro l'orizzonte, segnale della fine del lavoro per l'uomo di terra, è invece alba e segnale d'inizio, di nuovo giorno, per il pescatore:

È l'alba, per loro, questo tramonto, poi si fa notte, scura: la luna piena dice fame ai pescatori, offusca le lampare. Non sanno altro tempo fuori che questo, come le farfalle, i pipistrelli. ${ }^{31}$

È forse possibile leggere tale circolarità del tempo non tanto, o non solo, su un piano orizzontale, bidimensionale, come catena perenne di ritorni sempre uguali, quanto nella profondità, in verticale. Frequenti incursioni diacroniche paiono infatti restituire al presente uno specchio lontano, come se dalle profondità del passato e della memoria risalissero tracce, percorsi sommersi che emanano luce sul presente. Lo scorrere del tempo è ritratto come sovrapporsi di stratificazioni che, tagliate trasversalmente, svelano tra loro ricorrenze, similitudini, ritorni. È la lente della memoria e della storia che offre la terza dimensione alla lettura, e consente di cogliere e acuire il sentimento della frattura, della perdita, dei ritorni sotto nuove spoglie, della metamorfosi dell'identico.

L'«ordine continuo», il «rosario fatale della corsa» ${ }^{32}$ è dunque colto nell'accumulazione di rovine, su cui rinascono altri templi, altre illusioni:

La Contrada è illuminata da una luce livida. Si odono ululati di cani, pigolare d'uccelli notturni, mentre dall'alto, sulla fontana, sullo spiazzo, piovono lentamente falde di Luna simili a garze luminescenti. Così è stato e così sempre sarà: rovinano potenze, tramontano imperi regni civiltà, cadono astri, si

29. ID., Il sorriso dell'ignoto marinaio, Milano: Mondadori, 1976, p. 105.

30. Le pietre di Pantalica, p. 61.

31. La ferita dell'Aprile, p. 74.

32. Nottetempo..., p. 129. 
sfaldano, si spengono, uguale sorte hanno mitologie credenze religioni. Ogni fine è dolore, smarrimento ogni mutazione, stiamo saldi, pazienza, in altri teatri, su nuove illusioni nascono certezze. ${ }^{33}$

Quando il sole, reggitore dell'universale armonia, impallidisce nella sera, la melanconia attanaglia il Viceré di Lunaria («La luce solare, meridiana, che entra dai balconi, si smorza, da dorata diviene argentea, lunare, illividisce la sala, gli astanti.»). ${ }^{34}$

4.

Si apre qui una nuova possibile valenza della luminosità nell'opera consoliana, per indagare la quale è forse opportuno rifarsi al verso di Ungaretti che apre il capitolo Le pietre di Pantalica: «Soli andavamo dentro la rovina», da Ultimi cori per la terra promessa. I versi che precedono quello citato sono questi: "Calava a Siracusa senza luna / La notte e l'acqua plumbea / E ferma nel suo fosso riappariva, / Soli andavamo dentro la rovina / Un cordaro si mosse dal remoto». ${ }^{35}$ E così recita il primo paragrafo consoliano:

È l'ora in cui dal suo acuto ferino di bianca incandescenza si torce, si modula nei toni più mansueti, tangibili — oro arancio viola - la luce. Appaiono quindi le pietre, dal fitto fondale di pini e cipressi avanzano in linee parallele come onde, grigiorosa, muschiate, negli intervalli dove appena s'addensano sottilissime ombre, dentro il cerchio mistico dell'orchestra. Al cui centro, reale e ideale, bocca d'un segreto cunicolo, d'un buio ipogeo, è una porta, due corti pilastri e un architrave appena arcuato. Scenografia vera, come l'ha conciata degli scenografi il più riduttore, il più essenziale: il tempo. Nella cavea affollata, succede improvviso il silenzio. Ha inizio la rappresentazione della tragedia. ${ }^{36}$

Lo scenario di cerchi concentrici, come onde susseguentisi, la polarità verso un centro che ancora sfugge, perché imbocco nero verso profondità ipogee, l'accesso senza sbocco: è questa la scenografia del tempo, opera secolare di stratificazioni circolari i cui raggi ipotizzano un centro. L'architettura complessiva si svela nell'ora calda e lieve del tramonto, nella luce crepuscolare che segue "l'acuto ferino" del demone meridiano. Si tratta di un topos particolarmente caro a Ungaretti che al demone meridiano dedicò assidue ricerche, a partire dal commento alla leopardiana canzone Alla primavera. ${ }^{37}$

33. ID., Lunaria, Milano: Mondadori, 1985, p. 50.

34. Ibid., p. 139.

35. Cfr. Anche L'olivo e l'olivastro, p. 84.

36. Le pietre..., p. 157.

37. Nell'ora voraginosa, ora di «luce nera nelle vene», confluiscono memoria e malinconia, furore del sole e zenitale acedia, si congiungono demone meridiano e notturno meridio. Cfr. Carlo OssolA, «"Nell'abisso di sé”: Ungaretti e Racine», in B. M. DA RIF e C. GRIGGIO (a cura di), Dal Tommaseo ai contemporanei, Miscellanea di studi in onore di Marco Pecoraio, Firenze: Olschki Ed., 1991, p. 343-358. 


\section{Così scrive Consolo in Nottetempo:}

Venne poi il crepuscolo, la sera. Una sera azzurra e bruna, vermiglia e gialla. Con un reticolo d'ombre, di caligini, un turbine di braci. È l'ora questa degli scoramenti, delle inerzie, degli smarrimenti, delle malinconie senza rimedio, l'ora delle geometrie perfette, delle misure inesorabili, la sfera il compasso la clessidra la bilancia... (la luna suscita muffe, fiori di salnitro...) l'ora della luce bianca, della luce nera, sospesa e infinita. [...] Oltre sono le Rovine. ${ }^{38}$

Ritroviamo qui i termini ossimorici ungarettiani (la «luce nera nelle vene» di Ti svelerà) e in altri brani ancora immagini e allusioni al demone meridiano, notturno meridio. L'eccesso di luce, l'incandescenza meridiana, confondono confini, accecano, confluiscono nel buio, nell'inabissamento di sé, negli abissi di memoria, buio fluire del tempo. La trasfigurazione indotta dalla luce a perpendicolo è colta in più occasioni da Consolo; così nel Sorriso:

Luce che brucia, morde, divora lati spigoli contorni, stempera toni macchie, scolora. Impasta cespi, sbianca le ramaglie, oltre la piana mobile di scaglie orizzonti vanifica, rimescola le masse. ${ }^{39}$

o in alcune pagine di Nottetempo:

Quindi per gradi, per lenti processi discendiamo in spazi inusitati [...].

Ora, in questa luce nuova - privazione d'essa o luce stessa rovesciata, frantumo d'una lastra, rovinìo di superficie, sfondo infinito, abissitade — , in nuovi mondi o antichi, in luoghi ignoti risediamo. ${ }^{40}$

L'abbandono e il silenzio grevi dell'ora demente accompagnano e propiziano la calata nel vorticoso fluire del tempo, in un'immagine di memoria anche borgesiana: "d'un tempo che contiene tutti i tempi, un attimo ogni altro attimo. In quest' istante rapido, in quest' immensa stasi, l'uomo rivive tutta la sua vita». ${ }^{41}$ Ed ecco, calati nell'abisso, nel luogo di tutti i luoghi, ormai prossimi alla scaturigine, l'interrogativa sulla possibilità del racconto, memoria, scansione, parola, che è passaggio.

Ritorna l'immagine dell'accumulazione, del sovrapporsi di strati sopra strati, di lavorìo continuo, di movimento d'approssimazione incessante, ai confini del silenzio, come a strappare terre ai deserti. La risposta all'istanza finale è di desolazione siderale; nei "graffi indecifrati» si può sentire forse l'eco del «rilucere inveduto" ungarettiano (da Ultimi cori per la terra promessa: "Rilucere inveduto d'abbagliati / Spazi ove immemorabile / Vita passano gli astri / Dal peso pazzi della solitudine»). La vita è consumata ai margini del silenzio, è tra-

38. Nottetempo..., p. 65.

39. Il sorriso..., p. 17.

40. Nottetempo, p. 64

41. Ibid., p. 91. 
versata nel silenzio, silenzio come cerchio con il centro in ogni luogo e la circonferenza in nessuno:

E tu, e noi chi siamo? Figure emergenti o svanenti, palpiti, graffi indecifrati. Parola, sussurro, accenno, passo nel silenzio. ${ }^{42}$

I due protagonisti di Retablo si calano nelle acque lenitive dei Bagni Segestani, quasi alla ricerca di un Lete in cui adagiarsi e trovare ristoro. L'effetto del bagno induce alla perdita di sé nel "vacuo smemorante, nel vago vorticare». ${ }^{43}$

Il ricordo di Fabrizio Clerici va al primo incontro con Doña Teresita, un'apparizione nella luce e nello splendore; così Clerici riflette:

pur sulla soglia di questa forte terra, nel primo cerchio di questo vortice di luce, sull'ingresso di questo laberinto degli olezzi, nell'incamminamento di questa galleria de' singolari tratti e d'occhi ardenti, mi pare di viaggiare alla ricerca degli stampi o matrici del vostro maraviglioso sembiante. ${ }^{44}$

Se, da un lato, la visione dell'amata nella luce, il riscoprirne espressioni e gesti in altre figure femminili risponde a un topos letterario di tradizione antica, l'insistenza con cui paiono ricorrere i riferimenti alla lux veritatis, visione ineffabile del Vero, così come la non rara citazione di Platone anche attraverso la topica settecentesca della bellezza ideale ("eccelso modello di beltà») ${ }^{45}$ autorizzano un approfondimento dell'analisi delle valenze simboliche della luce anche in questa direzione.

Torniamo così al primo testo citato, 29 Aprile 1994: cronaca di una giornata, dove esplicito era il riferimento alla caverna platonica, in quel caso usato con vis polemica diretta contro il «regime telecratico» e le ingannevoli e false ombre televisive degne solo di una «degradata, miserabile caverna platonica». ${ }^{46}$ Nell'opera consoliana è possibile rintracciare con apprezzabile frequenza il riferimento ad una verità cui tornare suggerita da pallide tracce terrene, cui avvicinarsi procedendo per gradi, per accumulazioni, sempre vigili a non essere tratti in inganno da ombre fuggevoli, per arrivare preparati alla verità, in grado di sostenerne la tremenda forza autenticante. Così sin dalle prime prove:

Se mi si dice non si guarda alla finestra, allora mi volto alla parete bianca ed è più bello il gioco delle ombre rovesciate, e qualcuna l'indovino: il gobbo lo spazzino la posta il pane, l'ombra di tutti i giorni all'ore eguali. ${ }^{47}$

42. Ibid., p. 67.

43. Retablo, p. 61.

44. Ibid., p. 63.

45. Ibid., p. 64.

46. 29 aprile $1944 \ldots$, p. 7.

47. La ferita..., p. 81. 
La luce di verità è difficilmente sopportabile per occhi avvezzi all'ombra, ombra consolatoria di una luce che ferisce, come quella dei quadri caravaggeschi, in cui una lama di luce, proveniente dall'esterno, («La luce su Lucia giunge da fuori il quadro») ${ }^{48}$ ferisce il volto della figura. Caravaggio, "col suo corpaccio, la grossa testa bergamasca, i capelli peciosi e spessi, la fosca pelle, gli occhi ingrottati» pare posseduto da "dolore innominato» e da una «melanconia senza riparo che lo spingeva a denudare il mondo, togliere agli uomini, alle cose, ogni velame, ombra, illusione, esporli alla cruda lama della luce, alla spietata verità di questo giorno, di questa vita, squarcio, ferita immedicata, nel corpo della notte, del sonno, della stasi, amava scontrosamente la bellezza, pativa per la sua labilità, la sua assenza». ${ }^{49}$

Instabile sull'abisso e sul vuoto, si tende in funambolico equilibrio come "passo nel silenzio", ${ }^{50}$ come "ferita di luce nel buio" ${ }^{51}$ (G. Ungaretti, Immagini del Leopardi e nostre), la parola dolente.

\section{5.}

In conclusione, pare di poter affermare che la luce, le modulazioni o le assenze di luce, assumono nella scrittura consoliana valenze che chiaramente trascendono la mera descrittività, la nota paesaggistica o bozzettistica. Ho qui cercato di offrire alcune possibili chiavi interpretative. All'opposizione luceombra intesa come confronto tra Milano-città dei Lumi, meta per una Sicilia-cupa periferia di degrado, si accosta e sovrappone l'opposizione luce del passato e buio del presente. Questa seconda opposizione fa sì che i due poli, Milano e la Sicilia, che nella diatopia si oppongono e tra i quali si intraprende un movimento di perpetuo viaggio, di fughe e di ritorni eterni, confluiscano, in una prospettiva diacronica, a formare un unico punto, coincidano, condividendo il tramonto infinito del presente. La patria con cui riconciliarsi pare dunque sfuggire, risucchiata nel tempo, proiettata nel passato, luogo della memoria.

Tale opposizione, [buio-presente : luce-passato], non è resa solo attraverso il ricorso ad un "lessico della luce» per il passato e ad un «lessico dell'ombra» per il presente, ma anche mediante l'attribuzione alla luce di segni diversi: positivo in riferimento alla luce del passato e negativo in relazione alla luce del presente. In un paesaggio, umano e morale, armonico, come quello passato, la luce è forza vivificante, principio di movimento e di vita, di attività; nella miseria e nel degrado presenti la luce dissecca, prosciuga, svela silenzi, assenze.

Eppure la luce, pare leggere tra le righe o sous les mots, è sempre la stessa luce, da sempre scandisce il ritmo della giornata, dei lavori quotidiani, ma la cicli-

48. L'olivo..., p. 94.

49. Ibid., p. 88.

50. Nottetempo..., p. 67.

51. Giuseppe UnGARETTI, Immagini del Leopardi e nostre, saggio letto il 29 gennaio 1943 nell'Università di Roma, in M. Diacono e L. Rebay (a cura di), Saggi e interventi, Milano: Mondadori, 1974, p. 447. 
cità del tempo, i ritorni, non sono tra loro identici, si scrivono nella storia e la storia è sovrapposizione di strati, accumularsi di nuovi castelli su antiche rovine. Anche in questo caso dunque è la composizione di piano orizzontale e verticale, la discesa dal piano sincronico e orizzontale a quello della storia e della memoria, verticale, ad offrire nuove chiavi di lettura.

Il tempo della memoria e della malinconia è il tempo della luce nera in cui si congiungono demone meridiano e notturno meridio, di ungarettiana memoria. Ė il tempo di luce calcinante, ferale, e di buio vorticare nel flusso degli evi. Luce nera è dunque quella che accompagna la memoria e la malinconia, la ricerca e la catabasi, ed è luce bianca, luce dalla tremenda forza autenticante, la lux veritatis, ferita di luce che occhi avvezzi all'ombra non sanno sostenere. Ed è dunque infine anche monito alla vigilanza, alla diffidenza verso opachi lucori, finte luci, vacue illusioni e pericolose menzogne. 